$\mathrm{UBC} / \mathrm{S}-94 / 1$

hep-th/9410214

December 2, 2013

\title{
Critical Behaviour of a Fermionic Random Matrix Model at Large- $N$
}

\author{
Nicole Marshall[, Gordon W. Semenoff ${ }^{1}$ and Richard J. Szabo[ \\ Department of Physics \\ University of British Columbia \\ Vancouver, British Columbia, Canada V6T $1 Z 1$
}

\begin{abstract}
We study the large- $N$ limit of adjoint fermion one-matrix models. We find one-cut solutions of the loop equations for the correlators of these models and show that they exhibit third order phase transitions associated with $m$-th order multi-critical points with string susceptibility exponents $\gamma_{\text {str }}=-1 / m$. We also find critical points which can be interpreted as points of first order phase transitions, and we discuss the implications of this critical behaviour for the topological expansion of these matrix models.
\end{abstract}

\footnotetext{
${ }^{1}$ Work supported in part by the Natural Sciences and Engineering Research Council of Canada.

${ }^{2}$ Work supported in part by a University of British Columbia Graduate Fellowship.
} 
Hermitian matrix models are the classic example of a $D=0$ quantum field theory where 'tHooft's topological large- $N$ expansion [1] can be solved explicitly [2, 3]. They have recently been of interest in the study of the statistical mechanics of random surfaces [4] particularly for non-perturbative approaches to lower dimensional string theory [5]. There, the large- $N$ expansion coincides with the genus expansion and the large- $N$ limit exhibits phase transitions which correspond to the continuum limits of the discretized random surface theories [5]. Unitary matrix models also play a role in 2-dimensional QCD [6], mean-field computations in lattice gauge theory [7] and various other approaches to higher dimensional gauge theories such as induced QCD [8]. In this Letter we study a matrix model where the degrees of freedom are matrices whose elements are anticommuting Grassmann numbers. The partition function is

$$
Z=\int d \psi d \bar{\psi} \mathrm{e}^{N^{2}} \operatorname{tr} V(\bar{\psi} \psi)
$$

where $V$ is a polynomial potential,

$$
V(\bar{\psi} \psi)=\sum_{k=1}^{K} \frac{g_{k}}{k}(\bar{\psi} \psi)^{k}
$$

$\psi$ and $\bar{\psi}$ are independent $N \times N$ matrices with anticommuting nilpotent elements and the integration measure, $d \psi d \bar{\psi} \equiv \prod_{i, j} d \psi_{i j} d \bar{\psi}_{i j}$, is defined using the usual rules for integrating Grassmann variables, $\int d \psi_{i j} \psi_{i j}=1, \int d \psi_{i j} 1=0$. We normalize all traces here and in the following as $\operatorname{tr} V \equiv \frac{1}{N} \sum_{i} V_{i i}$.

Matrix models of this kind have been studied recently by Makeenko and Zarembo [9], and Ambjørn, Kristjansen and Makeenko [10]. They are motivated by models of induced gauge theories using adjoint matter where the Yang-Mills interactions of gluons are induced by loops with heavy adjoint scalar fields [ 8 or other kinds of matter such as heavy adjoint fermions [9, 11. Makeenko and Zarembo [9] have shown that the adjoint fermion matrix model (1) has many of the features of the more familiar Hermitian one-matrix model in the large- $N$ limit [5, 12], including multi-critical behaviour with a third order phase transition and string susceptibility with critical exponent $\gamma_{\text {str }}=-1 / m, m \in \mathbb{Z}^{+}$. They also showed that the loop equations for the model (1D) are identical to those for the Hermitian one-matrix model with generalized Penner potential [10, 13]

$$
Z_{P}=\int \prod_{i, j} d \phi_{i j} \mathrm{e}^{-N^{2} \operatorname{tr}(V(\phi)-2 \log \phi)}
$$

However, the loop equations for the two models should be solved with different boundary conditions and the solution beyond the leading order in large- $N$ is different in the two cases. It was argued in [9] and [10] that, as the Penner model corresponds to a certain statistical 
theory of triangulated random surfaces, the fermionic matrix model corresponds to a similar theory where the genus expansion has alternating signs. The resulting convergence of the sum over genera is reflected in the feature of the fermionic matrix model (1) that its partition function and observables are well-defined since the integrals over Grassmann variables always converge, in contrast to Hermitian matrix models [5, 12] where, typically, in the region of interest the integration over Hermitian matrices diverges (reflecting of course the divergence of the genus sum in the random surface model).

In fact, for any polynomial potential the integration over anticommuting variables in (11) can be formally performed by inserting the matrix-valued delta function $1=\int \prod_{i, j} d \phi_{i j} \delta(\phi-$ $\bar{\psi} \psi$ ), where $\phi$ is a Hermitian matrix, and using the identity

$$
\int \prod_{i, j} \frac{d \lambda_{i j}}{2 \pi} \int d \psi d \bar{\psi} \quad \mathrm{e}^{i \operatorname{tr} \lambda(\phi-\bar{\psi} \psi)}=\int \prod_{i, j} \frac{d \lambda_{i j}}{2 \pi} \operatorname{det}^{N}(-i \lambda) \mathrm{e}^{i \operatorname{tr} \lambda \phi}=\operatorname{det}^{N}\left(-\frac{\partial}{\partial \phi}\right) \delta(\phi)
$$

to obtain

$$
Z=\left.\operatorname{det}^{N}\left(\frac{\partial}{\partial \phi}\right) \mathrm{e}^{N^{2}} \operatorname{tr} V(\phi)\right|_{\phi=0}
$$

Similarly, any correlator is given by

$$
<\operatorname{tr}(\bar{\psi} \psi)^{p_{1}} \operatorname{tr}(\bar{\psi} \psi)^{p_{2}} \cdots>=\left.\frac{\operatorname{det}^{N}\left(\frac{\partial}{\partial \phi}\right) \operatorname{tr} \phi^{p_{1}} \operatorname{tr} \phi^{p_{2}} \cdots \mathrm{e}^{N^{2} \operatorname{tr} V(\phi)}}{\operatorname{det}^{N}\left(\frac{\partial}{\partial \phi}\right) \mathrm{e}^{N^{2} \operatorname{tr} V(\phi)}}\right|_{\phi=0}
$$

In spite of this good convergence of the partition function, it has been shown that in the infinite $N$ limit, the model has third order phase transitions [9, 10]. In this Letter, we shall examine the third order transitions in more detail and also argue that there may be a first order phase transition. We show that in a simple model with potential $V(\bar{\psi} \psi)=\bar{\psi} \psi+\frac{g}{3}(\bar{\psi} \psi)^{3}$ there are two critical points, one of them at the third order phase transition at $g=g_{c} \equiv 2 / 27$ which was studied in [9, 10] and the other at zero coupling $g=0$. In the region $g \in\left[0, g_{c}\right]$, multibranch one-cut solutions exist as well as a multi-cut solution of the matrix model whereas when $g \notin\left[0, g_{c}\right]$ a unique one-cut solution with real free energy exists.

At $g=g_{c}$ the unique one-cut solution for $g>g_{c}$ connects continuously with the one of the 3 one-cut solutions which has the minimal free energy in $0<g<g_{c}$. On the other hand, this solution is not continuous with the unique one-cut solution for $g<0$. The one-cut solution for $g<0$ connects to one which does not have minimal free energy. Ordinarily, the infinite energy barrier (the height of the barrier is of order $N^{2}$ ) between the metastable and stable one-cut solutions prevents tunneling and also a phase transition from occuring. However, if we restrict attention to one-cut solutions, and follow them over the range of $g$, we must encounter a discontinuity of the free energy somewhere, i.e. a first order phase transition. 
The choice of stable solution (the one-cut solution with minimum free energy) in the regime $g \in\left[0, g_{c}\right]$ results in a first order phase transition at $g=0$. With this stability requirement perturbation theory near the Gaussian point $g=0$ does not correctly reflect the properties of the theory when $g$ is small and negative. The critical point $g_{c}$ corresponds to the usual $m=2$ multi-critical point with $\gamma_{\text {str }}=-1 / 2$. In the following, we shall also explicitly construct onecut solutions for generic symmetric potentials and thus show how higher order multi-critical points can be realized in these models.

First, we shall discuss some general properties of the adjoint fermion matrix model (11). It possesses a continuous symmetry $\psi \rightarrow U \psi V^{-1}, \bar{\psi} \rightarrow V \bar{\psi} U^{-1}$ with $\{U, V\} \in G L(N, \mathbb{C}) \otimes$ $G L(N, \mathbb{C})$. In spite of this large degree of symmetry, it is not possible to diagonalize a matrix with anticommuting entries. Thus, unlike the more familiar Hermitian one-matrix models, the model (1) cannot be written as a statistical theory of eigenvalues. Nevertheless, in the large- $N$ limit it shares many of the properties of such a theory [2, [3]. Furthermore the large degree of symmetry restricts the observables to those which are essentially invariant functions of $\bar{\psi} \psi$. The chiral transformation $\psi \rightarrow \bar{\psi}, \bar{\psi} \rightarrow-\psi, \operatorname{tr}(\bar{\psi} \psi)^{k} \rightarrow(-1)^{k+1} \operatorname{tr}(\bar{\psi} \psi)^{k}$ is a symmetry when the potential is an odd polynomial. Furthermore, in that case, all even moments vanish, $<\operatorname{tr}(\bar{\psi} \psi)^{2 k}>=0$. This is the analog of a symmetric potential for a Hermitian matrix model [2].

In the large- $N$ limit, correlators of the matrix model factorize

$$
<\operatorname{tr} f(\bar{\psi} \psi) \operatorname{tr} g(\bar{\psi} \psi)>=<\operatorname{tr} f(\bar{\psi} \psi)><\operatorname{tr} g(\bar{\psi} \psi)>+\mathcal{O}\left(1 / N^{2}\right)
$$

This factorization property follows from the existence of a finite large- $N$ limit for the correlators $<\operatorname{tr}(\bar{\psi} \psi)^{k}>$ for arbitrary polynomial potential $V(\bar{\psi} \psi)=\sum_{k} \frac{g_{k}}{k}(\bar{\psi} \psi)^{k}$, since then the connected correlators are given by

$$
<\operatorname{tr}(\bar{\psi} \psi)^{p} \operatorname{tr}(\bar{\psi} \psi)^{k}>_{\mathrm{conn}}=\frac{1}{N^{2}} p \frac{\partial}{\partial g_{p}}<\operatorname{tr}(\bar{\psi} \psi)^{k}>\sim \frac{1}{N^{2}}
$$

Factorization and symmetry imply that the large- $N$ limit of the model is completely characterized by the set of correlators $<\operatorname{tr}(\bar{\psi} \psi)^{k}>$. Since the signs of the actions in (11) and (3) are opposite (from the different boundary conditions), it also follows that the connected correlators of the fermionic matrix model alternate in sign relative to those of the generalized Hermitian Penner model (3). It is this property that leads to an alternating series for the large- $N$ genus expansion of the fermionic matrix model (11).

When $N$ is finite, nilpotency of the components of $\psi$ and $\bar{\psi}$ implies that the moments $<\operatorname{tr}(\bar{\psi} \psi)^{k}>$ are non-zero only for $k \leq N^{2}$ and are therefore finite in number. In this case, 
the moment generating function

$$
\omega(z)=<\operatorname{tr} \frac{1}{z-\bar{\psi} \psi}>=\sum_{k=0}^{N^{2}}<\operatorname{tr}(\bar{\psi} \psi)^{k}>\frac{1}{z^{k+1}}
$$

has singularities only at the origin in the complex $z$-plane. The finite set of moments can always be obtained from a (not unique) distribution function $\rho$ with support in the complex plane

$$
<\operatorname{tr}(\bar{\psi} \psi)^{k}>=\int d \alpha \rho(\alpha) \alpha^{k} \quad \text { with } \quad \int d \alpha \rho(\alpha)=1
$$

The support of $\rho$ can be deduced from the position of the singularities of $\omega$ in (9). When $N$ and therefore the number of moments is finite the support of $\rho$ is concentrated near the origin

$$
\rho(\alpha)=\sum_{k=0}^{N^{2}} \frac{1}{k !}<\operatorname{tr}(\bar{\psi} \psi)^{k}>\left(-\frac{\partial}{\partial \alpha}\right)^{k} \delta(\alpha)
$$

In the large- $N$ limit, the spectral function $\rho(\alpha)$ can be a function with support on some contour in the complex plane. The distribution function $\rho$ is the analog in the fermionic matrix model of the density of eigenvalues in Hermitian one-matrix models as the quantity which specifies the solution of the model in the infinite $N$ limit [2].

The generating functions for the connected correlators are

$$
\omega_{n}\left(z_{1}, \ldots, z_{n}\right)=<\operatorname{tr} \frac{1}{z_{1}-\bar{\psi} \psi} \cdots \operatorname{tr} \frac{1}{z_{n}-\bar{\psi} \psi}>_{\mathrm{conn}}
$$

In particular, the loop correlator

$$
\omega_{1}(z) \equiv \omega(z)=\int d \alpha \frac{\rho(\alpha)}{z-\alpha}
$$

is analytic in $z$ away from the support of $\rho$ in the complex plane. The distribution function can be determined by computing the discontinuity of $\omega(z)$ across its support, where

$$
\omega\left(\lambda \pm \epsilon_{\perp}\right)=f d \alpha \frac{\rho(\alpha)}{\lambda-\alpha} \mp \frac{\epsilon_{\perp}}{\left|\epsilon_{\perp}\right|} \pi \rho(\lambda) \quad \text { for } \quad \lambda \in \operatorname{supp} \rho
$$

where $\epsilon_{\perp}(\lambda)$ is a complex number with infinitesimal amplitude and direction perpendicular to the integration contour at the point $\lambda$. The Schwinger-Dyson equation for the loop correlator $\omega(z)$ can be derived from the invariance of the partition function (四) under arbitrary changes of variables. It can be cast in the form [9, 10]

$$
\int d \psi d \bar{\psi} \frac{\partial}{\partial \psi_{i j}}\left[\left(\psi \frac{1}{z-\bar{\psi} \psi}\right)_{k \ell} \mathrm{e}^{N^{2} \operatorname{tr} V(\bar{\psi} \psi)}\right]=0
$$


In contrast to Hermitian matrix models [12, the identity (15) is exact for fermionic matrices. It leads to

$$
-z \omega(z)^{2}+\left(2-z V^{\prime}(z)\right) \omega(z)+V^{\prime}(z)+P(z)=z \omega_{2}(z, z)
$$

where $P(z)$ is a polynomial of degree $K-2$

$$
P(z)=\sum_{k=2}^{K} g_{k} \sum_{p=0}^{k-2}<\operatorname{tr}(\bar{\psi} \psi)^{k-1-p}>z^{p}
$$

Factorization implies that the connected correlators are all suppressed by factors of $1 / N^{2}$ and the term on the right-hand side of the loop equation (16) vanishes in the large- $N$ limit. Then the loop equation has solution

$$
\omega(z)=\frac{1}{z}-\frac{V^{\prime}(z)}{2}+\frac{1}{z} \sqrt{1+\left(\frac{z V^{\prime}(z)}{2}\right)^{2}+z P(z)}
$$

where the sign of the square root is chosen to yield the correct asymptotic behavior $\omega(z) \rightarrow 1 / z$ at $|z| \rightarrow \infty$. The branches of the square root must be placed so that it is negative near the origin in order to cancel the pole at $z=0$. If the potential is a polynomial of order $K$, the solution will in general possess a square root singularity with $K$ branch cuts and the spectral density $\rho$ will have $K$ contours in its support.

The simplest solution of the model is the one-cut solution which assumes that the singularities of $\omega(z)$ consist of only a single square root branch cut, so that the distribution $\rho$ has support only on one arc in the complex plane with endpoints at some complex values $a_{1}$ and $a_{2}$. This solution for $\omega(z)$ can be represented in the form [9, 10]

$$
\omega(z)=\oint_{\mathcal{C}} \frac{d w}{4 \pi i} \frac{V^{\prime}(w)-2 / w}{z-w} \sqrt{\frac{\left(z-a_{1}\right)\left(z-a_{2}\right)}{\left(w-a_{1}\right)\left(w-a_{2}\right)}}
$$

where the closed contour $\mathcal{C}$ encloses the support of the spectral function but not the point $w=z$. The endpoints of the cut can then be found by imposing the asymptotic boundary condition $\omega(z) \rightarrow 1 / z$ at $|z| \rightarrow \infty$ on the solution ([19), which leads to the two equations

$$
\oint_{\mathcal{C}} \frac{d w}{2 \pi i} \frac{V^{\prime}(w)-2 / w}{\sqrt{\left(w-a_{1}\right)\left(w-a_{2}\right)}}=0 \quad, \quad \oint_{\mathcal{C}} \frac{d w}{2 \pi i} \frac{w V^{\prime}(w)-2}{\sqrt{\left(w-a_{1}\right)\left(w-a_{2}\right)}}=2
$$

To determine the precise location of the support contour of $\rho$ in the complex plane, we first use the observation of [9] that the large- $N$ equation (16) for the loop correlator is identical to the loop equation for the generalized Penner model (3). In the large- $N$ limit, the spectral density therefore obeys the saddle-point equation [2]

$$
\frac{2 / \lambda-V^{\prime}(\lambda)}{2}=f d \alpha \frac{\rho(\alpha)}{\lambda-\alpha} \quad, \quad \lambda \in \operatorname{supp} \rho
$$


Note that this equation can be obtained from the discontinuity (14) of the loop correlator (18), and it also follows from the minimization condition for the free energy

$$
F=\lim _{N \rightarrow \infty} \frac{1}{N^{2}} \log Z_{P}=\int d \alpha \rho(\alpha)(V(\alpha)-2 \log \alpha)+\int f d \alpha d \lambda \rho(\alpha) \rho(\lambda) \log (\alpha-\lambda)
$$

with respect to the distribution function $\rho$. Note the change in sign of the fermionic free energy relative to the Hermitian case. The double integral in (22) is evaluated by integrating up the saddle-point equation (21). This introduces a logarithmic divergence at $\lambda=0$ arising from the Penner potential in (3) which we remove by subtracting from (22) the Gaussian free energy $F_{G}$ defined by setting $g_{k}=0$ for $k>1$ in (22)

$$
F-F_{G}=\frac{1}{2} \int d \alpha \rho(\alpha)(V(\alpha)-2 \log \alpha)+f d \alpha \rho(\alpha) \log \alpha
$$

where we have ignored terms independent of $g_{k}, k>1$. The support contour of $\rho$ can be determined from the David primitive function [14]

$$
G(w)=\int_{a_{1}}^{w} d z\left(\frac{2}{z}-V^{\prime}(z)-2 \omega(z)\right)
$$

The support of $\rho$ is an arc connecting $a_{1}$ to $a_{2}$ in the complex plane along which $G(w)$ is purely imaginary and which can be embedded in a region where $\operatorname{Re} G(w)<0$ [14].

For illustration, we shall consider the cubic potential

$$
V(z)=t z+\frac{g}{3} z^{3}
$$

for which

$$
\omega(z)=\frac{1}{z}-\frac{t}{2}-\frac{g z^{2}}{2}+\frac{1}{2 z} \sqrt{g^{2} z^{6}+2 \operatorname{tg} z^{4}+\left(t^{2}+4 g \xi\right) z^{2}+4}
$$

where $\xi$ is the as yet unknown correlator $\xi=<\operatorname{tr} \bar{\psi} \psi>$. In this case the vanishing of all even moments, $\int d \alpha \rho(\alpha) \alpha^{2 k}=0$, implies that the endpoints of the support contour of the continuous function $\rho$ lie in the complex plane and are symmetric on reflection through the origin. Furthermore, an application of Wick's theorem shows that the series (9) in the odd moments is alternating.

Generically the square root in $\omega(z)$ has three branch cuts, so that in the general case the distribution $\rho$ will have three disjoint and symmetric (about the origin) support contours. The one-cut solution for (26) takes the form

$$
\omega(z)=\frac{1}{z}-\frac{t}{2}-\frac{g z^{2}}{2}+\frac{g z^{2} \pm b}{2 z} \sqrt{z^{2}+4 / b^{2}}
$$

where comparing the polynomial coefficients in (27) with those of (26) shows that the parameter $b$ and the correlator $\xi$ are determined by the two equations

$$
\pm b^{3}-t b^{2}+2 g=0
$$




$$
b^{3}-\left(t^{2}+4 g \xi\right) b \pm 8 g=0
$$

The sign ambiguity here can be eliminated by requiring that at $g=0$ the correct Gaussian value $b(g=0, t)=t$ for $b$ [9] be attainable. This is the boundary condition that is relevant for an interpretation of this matrix model as a discretized random surface theory, i.e. for a consistent perturbative expansion of the model in the coupling constant $g$. It means that we take the positive sign in the above equations. The choice of negative sign yields solutions with boundary conditions at $g=0$ appropriate to generalized Penner models [10, 13]. The equation (28) and this sign ambiguity also follow from the contour integrals (20).

We assume henceforth that $t$ is a positive constant. The 3 solutions of (28) are

$$
\begin{gathered}
b_{0}(x, t)=\frac{t}{3}\left(\beta^{1 / 3}(x)+\beta^{-1 / 3}(x)+1\right) \\
b_{ \pm}(x, t)=\frac{t-b_{0}(x, t)}{2} \pm \frac{i \sqrt{3} t}{6}\left(\beta^{1 / 3}(x)-\beta^{-1 / 3}(x)\right)
\end{gathered}
$$

where

$$
\beta(x)=2 x-1+2 \sqrt{x(x-1)}
$$

and we have introduced the scaling parameter $x=1-\frac{27 g}{2 t^{3}}$. When $x \leq 0\left(g \geq g_{c} \equiv \frac{2 t^{3}}{27}\right)$ or $x \geq 1(g \leq 0), \beta(x)$ is a monotone real-valued function with $\beta(x) \geq 1$ for $x \geq 1$ and $\beta(x) \leq-1$ for $x \leq 0$. In the region $0<x<1\left(0<g<g_{c}\right), \beta(x)$ is a complex-valued function with unit modulus. The function (30) is always real-valued and the region $0<x<1$ is the region wherein all 3 roots (30), (31) of the cubic equation (28) are real. These 3 roots can all be obtained from (30) by choosing the 3 inequivalent cube roots of $\beta(x)$. For $x \notin(0,1)$ the solutions (31) are complex. For the fermionic matrix model, where the distribution function $\rho$ can be complex-valued, there is no immediate reason to disregard complex-valued endpoints for the support of $\rho$. However, the free energy (23) for the cubic potential (25) up to terms independent of $b$ and $g$ is

$$
F(x, t)-F_{G}(t)=\frac{t(3 b(x, t)-t)}{6 b^{2}(x, t)}
$$

where we have used the spectral density determined by (14) and (27)

$$
\rho(\alpha)=\frac{1}{2 \pi i}\left(b+g \alpha^{2}\right) \sqrt{1+\frac{4}{b^{2} \alpha^{2}}} \quad ; \quad \alpha \in \mathcal{C}_{b}
$$

where $\mathcal{C}_{b}=\operatorname{supp} \rho$ and $b(g, t)$ are given by (30) and (31). It is immediately seen that the free energy (33) is complex-valued for the values (31) of $b(x, t)$ for $x \notin(0,1)$. Such a free energy leads to an unstable state and we therefore consider only the real-valued solutions to (28). 
The support contour $\mathcal{C}_{b}$ on which (34) is defined is found from the David function (24) which is

$$
\begin{array}{r}
G(z)=-\sqrt{b^{2} z^{2}+4}-\operatorname{sgn}(b) \log \left(\frac{\sqrt{b^{2} z^{2}+4}-2}{\sqrt{b^{2} z^{2}+4}+2}\right)-\frac{g}{3|b|^{3}}\left(b^{2} z^{2}+4\right)^{3 / 2} \\
+\frac{g}{b^{2}} \log \left[\frac{i}{2}\left(\sqrt{b^{2} z^{2}+4}+|b| z\right)\right]-i \pi \operatorname{sgn} b
\end{array}
$$

where the branch of the square roots in (35) is taken to be the straight line joining the points $\pm 2 i /|b|$. A careful examination of the equation $\operatorname{Re} G(z)=0$ and of the region where $\operatorname{Re} G(z)<0$ shows that the contour $\mathcal{C}_{b}$ cannot cross the imaginary axis for $|\operatorname{Im} z|>2 /|b|$ and that it crosses the real axis at some non-zero values of order $\pm 1 /|b|$. The regions $\operatorname{Re} G(z)<0$ are to the right of these crossing points (but note that $\operatorname{Re} G(z)$ changes sign across $\mathcal{C}_{b}$ ). Thus the contour $\mathcal{C}_{b}$ in (34) can be taken as the counterclockwise oriented half-circle of radius $2 /|b|$ in the first and fourth quadrants of the complex $\alpha$-plane. It is easy to verify that with this definition of $\rho$ the equations (10) and (13) are satisfied, as is (29) from evaluating the correlator $\xi=\int d \alpha \rho(\alpha) \alpha$ with this distribution function.

There are 2 critical points in this large- $N$ matrix model, at $g=0$ and $g=g_{c}$, which separate 3 phases determined by the analytic structure of the function (32), i.e. the one-cut solution is a non-analytic function of $x$ about $x=0$ and $x=1$ where it acquires a square root branch cut. For $x \geq 1$ the solution

$$
b_{0}(x, t)=\frac{t}{3}\left[1+(2 x-1+2 \sqrt{x(x-1)})^{1 / 3}+(2 x-1+2 \sqrt{x(x-1)})^{-1 / 3}\right], x \geq 1
$$

of (28) satisfies the Gaussian boundary condition $b_{0}(x=1, t)=t$. When $x \leq 0$ the real solution for $b$ is

$$
b_{0}(x, t)=\frac{t}{3}\left[1-|2 x-1+2 \sqrt{x(x-1)}|^{1 / 3}-|2 x-1+\sqrt{x(x-1)}|^{-1 / 3}\right], x \leq 0
$$

As $x$ is varied between 0 and $1, \beta(x)$ has modulus one and phase which varies from $\pi$ to $0: \beta(x)=\mathrm{e}^{i \phi(x)}$ where

$$
\phi(x)=\arctan \left(\frac{2 \sqrt{x(1-x)}}{2 x-1}\right) \in[0, \pi]
$$

The arctangent function in (38) is well-defined only up to an integral multiple of $2 \pi$, and the three real solutions for $b$ are

$$
b(x, t)=\frac{t}{3}\left[1+2 \cos \left\{\frac{1}{3} \arctan \left(\frac{2 \sqrt{x(1-x)}}{2 x-1}\right)+\frac{2 n \pi}{3}\right\}\right] \quad ; \quad 0<x<1, n=0,1,2
$$


The branch which matches (37) is the one with $n=1$, whereas the branch which matches (36) is the one with $n=0$. The branch with $n=2$ does not connect with either solution. Any of these 3 branches can be used to define the one-cut solution (27). The free energy (33) is positive for all $x \in(0,1)$ for the $n=0$ branch, negative for all $x \in(0,1)$ for the $n=1$ branch, and for the $n=2$ branch it is positive for $0<x<\frac{1}{2}$ and flips sign for the rest of the interval at $x=\frac{1}{2}$. The $n=1$ branch in (39) is the ground state solution in the region $0<x<1$.

The free energy associated with this stable one-cut solution is discontinuous across $g=0$, and thus with this choice of branch in the regime $0<x<1$ the Gaussian point of this matrix model is a critical point of a first order phase transition. The other one-cut solution which is a perturbation of the Gaussian solution is metastable but can still be thought of as a valid solution of the model since the barrier is infinite at $N=\infty$. This is similar to the situation in the Hermitian one-matrix model with symmetric polynomial potential of degree 6 [15]. There a phase transition occurs due to an infinite volume effect, as opposed to a large- $N$ effect where the only possibilities could be second or third order phase transitions. There is also the possibility that the loop correlator (26) evolves into a three-cut phase at $g=0$, corresponding to a third order phase transition at $g=0$ (see below), but there is no immediate indication of this since in the fermionic case the spectral measure $\rho(\alpha) d \alpha$ need not be positive. This possibility is also suggested by the exact form (26) of the loop correlator: Although the onecut ansatz (27) is insensitive to a change in sign of $g$, the analytic properties of (26) might be affected by the passage through $g=0$.

The existence of 3 phases in this matrix model and the possibility of a first order phase transition at the Gaussian point $g=0$ are completely unlike what occurs in the conventional polynomial Hermitian matrix models [5, 12] or in Penner models [10, [16]. The unusual phase transition at $g=0$ would imply that the consistent perturbative expansion of the theory in $g$ has a preferred direction through values of $g$ with a definite sign (corresponding to $-\operatorname{sgn} t$ ). This fact is important for the interpretation of the fermionic one-matrix model as a statistical theory of discretized random surfaces. Notice, however, that the free energy (33) with the choice of stable branch for $x \in(0,1)$ is continuous across the point $g=g_{c}$ which coincides with the critical point found in [10].

The scaling behaviour of the matrix model in the vicinity of its critical points is determined by the string susceptibility

$$
\chi(g, t)=-\frac{1}{N^{2}} \frac{\partial^{2} \log Z}{\partial g^{2}}=-\frac{1}{3} \frac{\partial}{\partial g}<\operatorname{tr}(\bar{\psi} \psi)^{3}>
$$

The critical exponent $\gamma_{\text {str }}^{(i)}$ at each critical point $g_{c}^{(i)}$ is defined by the leading non-analytic 
behaviour of (40) [5]

$$
\chi(g, t) \sim_{S}\left(g-g_{c}^{(i)}\right)^{-\gamma_{\mathrm{str}}^{(i)}} \quad \text { as } \quad g \rightarrow g_{c}^{(i)}
$$

where $\sim_{S}$ denotes the most singular part of the function in a neighbourhood of the critical point. In terms of the scaling variable $x$, the susceptibility (40) is

$$
\chi(x, t)=\frac{972}{t^{6}\left(\beta^{2 / 3}+\beta^{1 / 3}+1\right)^{6}}\left[1-8 x+8 x^{2}+4 \sqrt{\frac{x}{x-1}}\left(1-3 x+2 x^{2}\right)\right]
$$

From (42) we find that the leading singular parts of the susceptibility near each of the two critical points $g=g_{c}$ and $g=0$ are respectively

$$
\begin{gathered}
\chi(x, t) \sim_{S}-\frac{15552}{t^{6}} \sqrt{x} \quad \text { as } \quad x \rightarrow 0 \\
\chi(x, t) \sim_{S} \frac{11648}{3 t^{6}} \sqrt{x-1} \text { as } x \rightarrow 1
\end{gathered}
$$

Both critical points therefore have string constant $\gamma_{\text {str }}=-1 / 2$ which coincides with those of the usual genus zero $m=2$ quantum gravity models [12].

In particular, this shows that with the choice of stable branch for $x \in(0,1)$ the phase transition at the non-zero critical coupling $g=g_{c}$ is of third order. Indeed, the critical point $g=g_{c}$ enjoys all of the properties of a conventional $m=2$ multi-critical point [12]. The information relevant to the topological genus expansion in string theory is contained in this non-zero third order critical coupling. As argued in [10, this $m=2$ multi-critical point results in a genus expansion which is an alternating series but otherwise coincides with the usual Painlevé expansion [5].

Thus the 2 critical points of the fermionic one-matrix model, which arise as those points in parameter space where the cubic equation (28) which determines the one-cut solution acquires a double real root, provide the necessary information for the perturbative and topological expansions of the theory. In the 2 phases outside of the region $0<x<1$ the one-cut solution is unique, while in the phase $x \in(0,1)$ a three-cut solution can in addition exist. The scaling behaviours (43) and (44) indicate that the 2 transitions into the multi-cut phase would both be of third order, while the transitions into the stable one-cut phase are of first and third order. The existence of a single-cut or multi-cut phase in $0<x<1$ is determined by which one of these 2 possibilities is in fact the vacuum state. The phase structure of this model is reminiscent of the triple point of a liquid-vapour-solid system, where there is a classical forbidden region in the liquid phase for the isotherms to pass through. The system can however supercool from the vapour to solid phase by tunneling through this region which corresponds to a first order phase transition, while the other phase transitions are of third order. In our case this "forbidden" region is $0<x<1$ (as this is where the function $\beta(x)$ 
becomes non-analytic) and it has width $g_{c}=2 t^{3} / 27$. The triple point where all 3 phases coexist is then obtained by letting $t \rightarrow 0$.

We conclude by discussing the critical behaviour associated with higher order potentials. For simplicity we consider the chirally symmetric case where the potential (2) is a generic odd polynomial, i.e. $g_{2 k}=0$ for all $k$ in (2), with $K=\operatorname{deg} V>3$ an odd integer. The solution for the loop correlator is then

$\omega(z)=\frac{1}{z}-\sum_{k=1}^{\frac{K+1}{2}} \frac{g_{2 k-1}}{2} z^{2(k-1)}+\frac{1}{2 z}\left(4+\sum_{k, m=1}^{\frac{K+1}{2}} g_{2 k-1} g_{2 m-1} z^{2(k+m-1)}+\sum_{k, m=1}^{m+k \leq \frac{K+1}{2}} g_{2(k+m)-1} \xi_{2 m} z^{2 k}\right)^{1 / 2}$

where $\xi_{2 m}$ are the as yet unknown moments $\xi_{2 m}=<\operatorname{tr}(\bar{\psi} \psi)^{2 m-1}>$. The one-cut solution for (45) takes the form

$$
\omega(z)=\frac{1}{z}-\sum_{k=1}^{\frac{K+1}{2}} \frac{g_{2 k-1}}{2} z^{2(k-1)}+\frac{1}{2 z}\left(g_{K} z^{K-1}+\sum_{k=1}^{\frac{K-3}{2}} a_{2 k} z^{2 k}+b\right) \sqrt{z^{2}+4 / b^{2}}
$$

where we have fixed the sign in front of the parameter $b$ by the same convention as before. The one-cut ansatz (46) along with the general solution (45) together involve $K-1$ unknown parameters $-b$, the $(K-3) / 2$ polynomial coefficients $a_{2 k}$, and the $(K-1) / 2$ correlators $\xi_{2 k}$.

These parameters can be found by comparing the various polynomial coefficients of (45) with those of (46), which leads to the set of equations

$$
\begin{gathered}
b^{3}+8 a_{2}-b\left(g_{1}^{2}+\sum_{k=1}^{\frac{K-1}{2}} g_{2 k+1} \xi_{2 k}\right)=0 \quad, \quad 2 b^{3}+8 b a_{4}+4 a_{2}^{2}-b^{2}\left(2 g_{1} g_{3}+\sum_{k=1}^{\frac{K-3}{2}} g_{2 k+3} \xi_{2 k}\right)=0 \\
2 b g_{K} a_{K-5}+8 a_{K-3}-b \sum_{k=1}^{K-2} g_{2 k-1} g_{2 K-2 k-3}=0 \quad, \quad 2 b^{2} g_{K} a_{K-3}+4 g_{K}^{2}-b^{2} \sum_{k=1}^{K-1} g_{2 k-1} g_{2 K-2 k-1}=0 \\
2 b^{2} a_{K-3}+8 g_{K}-b\left(g_{K} \xi+\sum_{k=1}^{\frac{K-1}{2}} g_{2 k-1} g_{K-2 k}\right)=0 \quad, \quad 2 b^{3} g_{K}+8 b g_{K} a_{2}-b^{2} \sum_{k=1}^{\frac{K+1}{2}} g_{2 k-1} g_{K-2 k+2}=0
\end{gathered}
$$

When $K>7$ we have in addition the sets of equations

$$
\begin{array}{r}
2 b^{3} a_{2(m-1)}+8 b a_{2 m}+4 a_{2} a_{2(m-1)}+\sum_{k=1}^{m-2}\left(b^{2} a_{2 k} a_{2(m-k-1)}+4 a_{2 k} a_{2(m-k)}\right) \\
-b^{2}\left(\sum_{k=1}^{m} g_{2 k-1} g_{2(m-k)+1}+\sum_{k=1}^{\frac{K+1-2 m}{2}} g_{2(m+k)-1} \xi_{2 k}\right)=0
\end{array}
$$


for $3 \leq m \leq \frac{K-3}{2}$, and

$$
\begin{array}{r}
2 b^{2} g_{K} a_{2 m-K-1}+8 b a_{2 m-K+1}+b^{2} \sum_{k=1}^{m-2} a_{2 k} a_{2(m-k-1)} \\
+4 \sum_{k=1}^{m-1} a_{2 k} a_{2(m-k)}-b^{2} \sum_{k=1}^{m} g_{2 k-1} g_{2(m-k)+1}=0
\end{array}
$$

for $\frac{K+3}{2} \leq m \leq K-3$.

(47)-(51) yield a complete set of equations for the $K-1$ unknown coefficients of the onecut solution (46) in terms of the coupling constants of the potential (2). The parameter $b$ can alternatively be found from the contour integrals (20) which lead to a $K$-th order equation for $b$

$$
b^{K}+\sum_{k=1}^{\frac{K+1}{2}} \frac{(-1)^{k} 2^{k-1}(2 k-3) ! !}{(k-1) !} g_{2 k-1} b^{K-2 k+1}=0
$$

Since $K$ is odd this equation always has a real solution, and as before the one-cut solution can always be constructed. The spectral density is

$$
\rho(\alpha)=\frac{1}{2 \pi i}\left(g_{K} \alpha^{K-1}+\sum_{k=1}^{\frac{K-3}{2}} a_{2 k} \alpha^{2 k}\right) \sqrt{1+\frac{4}{b^{2} \alpha^{2}}} \quad ; \quad \alpha \in \mathcal{C}_{b}
$$

The first $(K-1) / 2$ moments of this distribution are given by the solutions to (47)-(51).

In general (52) will acquire multiple real roots at some coupling $g_{2 k-1}^{c}$ which will be a critical point of a third order phase transition with string constant $\gamma_{\text {str }}=-1 / 2$. We expect that the phase with multiple real roots will be bounded by other coupling constant values so that the model will contain several critical points corresponding possibly to different order phase transitions. These critical points might provide information about this matrix model other than that relevant to the genus expansion. Notice that since the potential now depends on more parameters, we can adjust them in such a way that $m-1$ regular zeroes of (53) coalesce with a cut end-point $\pm 2 i /|b|$ at criticality for the same critical coupling $g_{2 k-1}^{c} \cdot g_{2 k-1}^{c}$ will then be an $m$-th order multi-critical point with susceptibility exponent $\gamma_{\text {str }}=-1 / m$ [12]. In this way the construction above shows explicitly how more general two-dimensional quantum gravity theories [5, 12] may be obtained from continuum limits of fermionic matrix models.

The authors gratefully thank Yu. Makeenko for helpful discussions and comments on the manuscript. We also thank L. Paniak for helpful discussions. 


\section{References}

[1] G. 'tHooft, Nucl. Phys. B72 (1974), 461.

[2] E. Brézin, C. Itzykson, G. Parisi and J.-B. Zuber, Commun. Math. Phys. 59 (1978), 35.

[3] D. Bessis, C. Itzykson and J.-B. Zuber, Adv. Appl. Math. 1 (1980), 109.

[4] J. Ambjørn, B. Durhuus and J. Fröhlich, Nucl. Phys. B257 [FS14] (1985), 433; F. David, Nucl. Phys. B257 [FS14] (1985), 45; V. A. Kazakov, Phys. Lett. B150 (1985), 282; V. A. Kazakov, I. K. Kostov and A. A. Migdal, Phys. Lett. B157 (1985), 295.

[5] E. Brézin and V. A. Kazakov, Phys. Lett. B236 (1990), 144; M. R. Douglas and S. H. Shenker, Nucl. Phys. B335 (1990), 635; D. J. Gross and A. A. Migdal, Phys. Rev. Lett. 64 (1990), 127.

[6] D. J. Gross and E. Witten, Phys. Rev. D21 (1980), 446.

[7] A. Drouffe and J.-B. Zuber, Phys. Rep. 102 (1983), 1.

[8] V. A. Kazakov and A. A. Migdal, Nucl. Phys. B397 (1993), 214.

[9] Yu. Makeenko and K. Zarembo, Nucl. Phys. B422 (1994), 237.

[10] J. Ambjørn, C. F. Kristjansen and Yu. Makeenko: Generalized Penner Models to all Genera, Niels Bohr Institute preprint NBI-HE-94-14 (1994).

[11] S. Khokhlachev and Yu. Makeenko, Mod. Phys. Lett. A7 (1992), 3653; A. A. Migdal, Mod. Phys. Lett. A8 (1993), 359.

[12] V. A. Kazakov, Mod. Phys. Lett. A4 (1989), 2125.

[13] R. C. Penner, Commun. Math. Phys. 113 (1987), 299; J. Diff. Geom. 27 (1988), 35.

[14] F. David, Nucl. Phys. B348 (1991), 507.

[15] J. Jurkiewicz, Phys. Lett. B245 (1990), 178.

[16] S. Chaudhuri, H. M. Dykstra and J. D. Lykken, Mod. Phys. Lett. A6 (1991), 1665. 